\title{
Lathyranone A: A Diterpenoid Possessing an Unprecedented Skeleton from Euphorbia lathyris
}

Suo Gao, ${ }^{+, \S}$ Hai-Yang Liu, ${ }^{+}$Yue-Hu Wang, ${ }^{+}$Hong-Ping He, ${ }^{+}$Jun-Song Wang, ${ }^{++}$

Ying-Tong Di, ${ }^{+}$Chun-Shun Li, ${ }^{+}$Xin-Fang, ${ }^{+}$Xiao-jiang Hao, ${ }^{*},+$

State Key Laboratory of Phytochemistry and Plant Resources in West China, Kunming Institute of Botany, Chinese Academy of Sciences, Kunming 650204,Yunnan, P.R. China, and Graduate School of Chinese Academy of Sciences, Beijing 100039, P.R. China, German Cancer Research Centre, Pharmaceutical Biology (C015),

\section{Supporting Information}

S1. title of manuscript and author list.

S2 $1 \mathrm{H}$ spectrum of compound.

S3 C13 and DEPT spectrum of compound

S4 HSQC spectrum of compound 1

S5 HMBC spectrum of compound 1

S6 1H-1H COSY spectrum of compound 1

S7 ROESY spectrum of compound 1 
$1 \mathrm{H}$ spectrum of compound 1

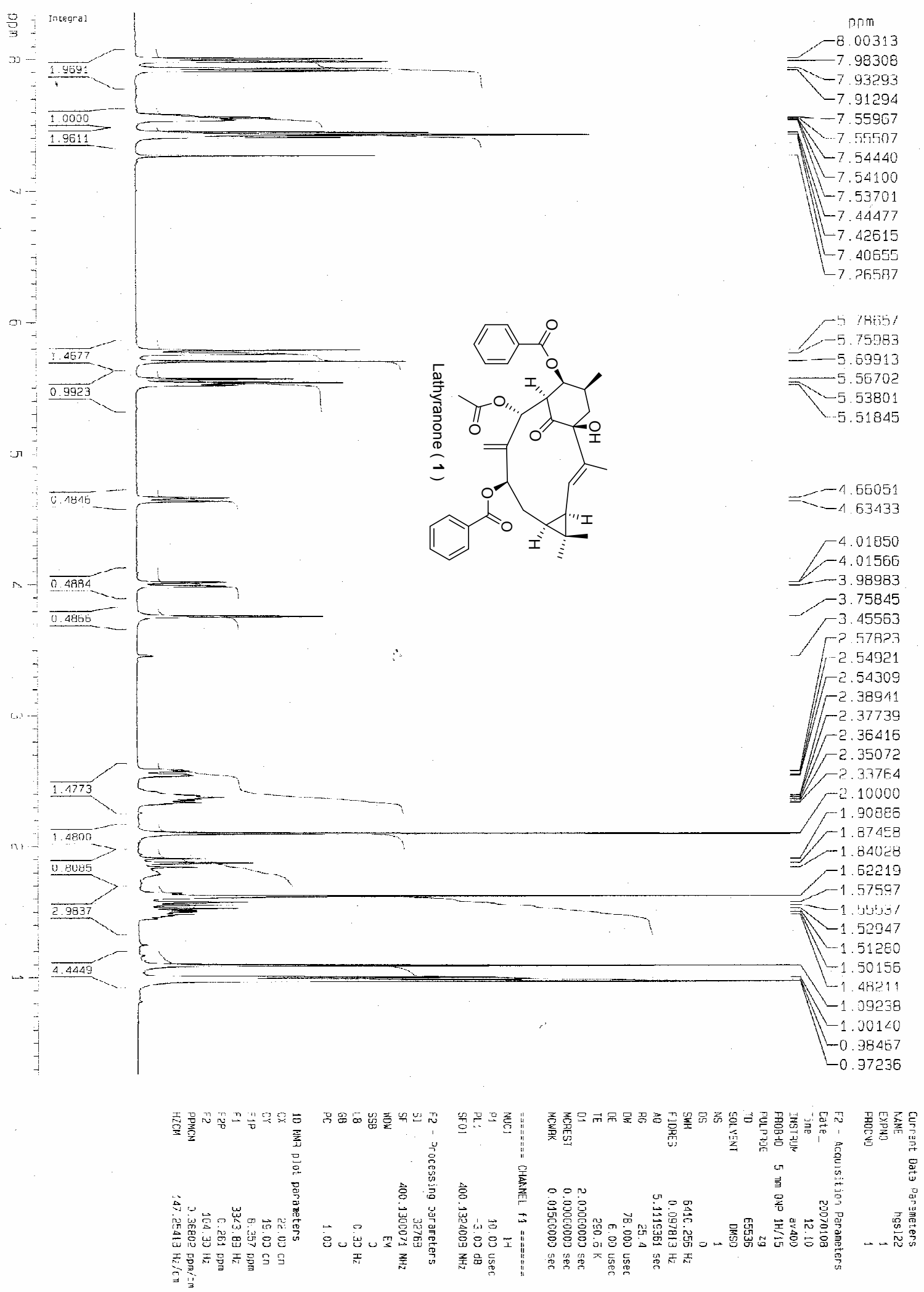


C13 and DEPT spectrum of compound 1

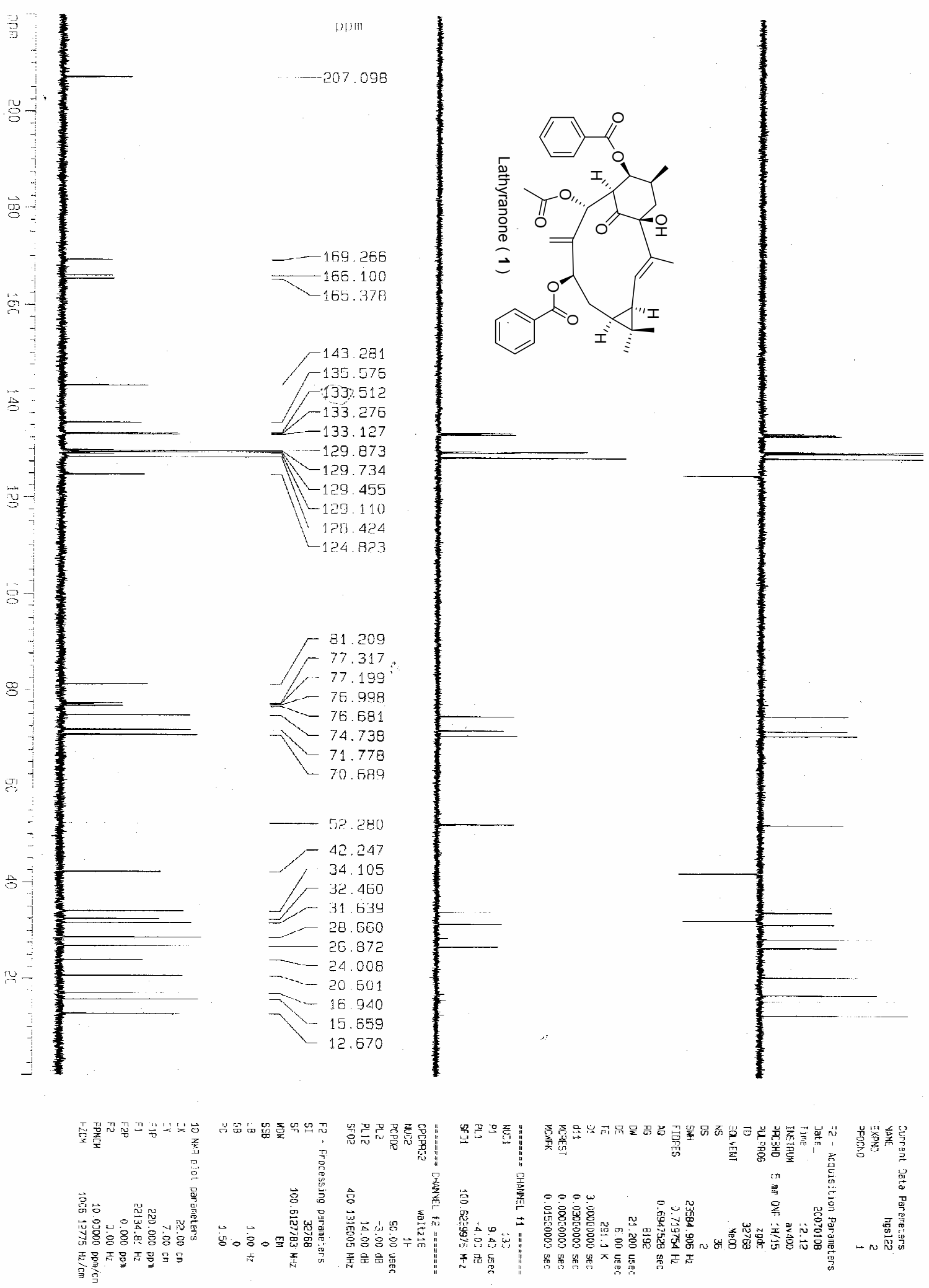


HSQC spectrum of compound 1
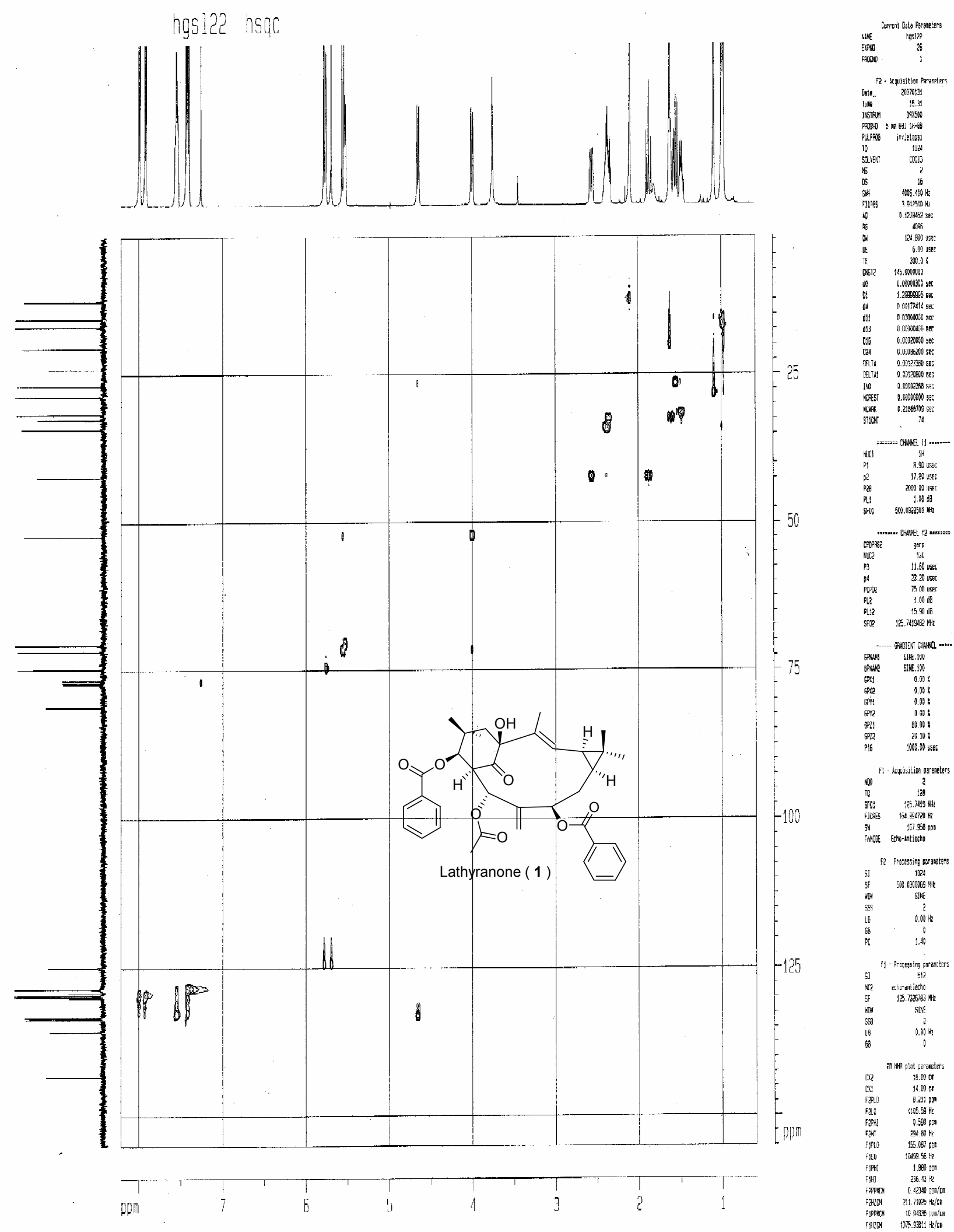
HMBC spectrum of compound 1

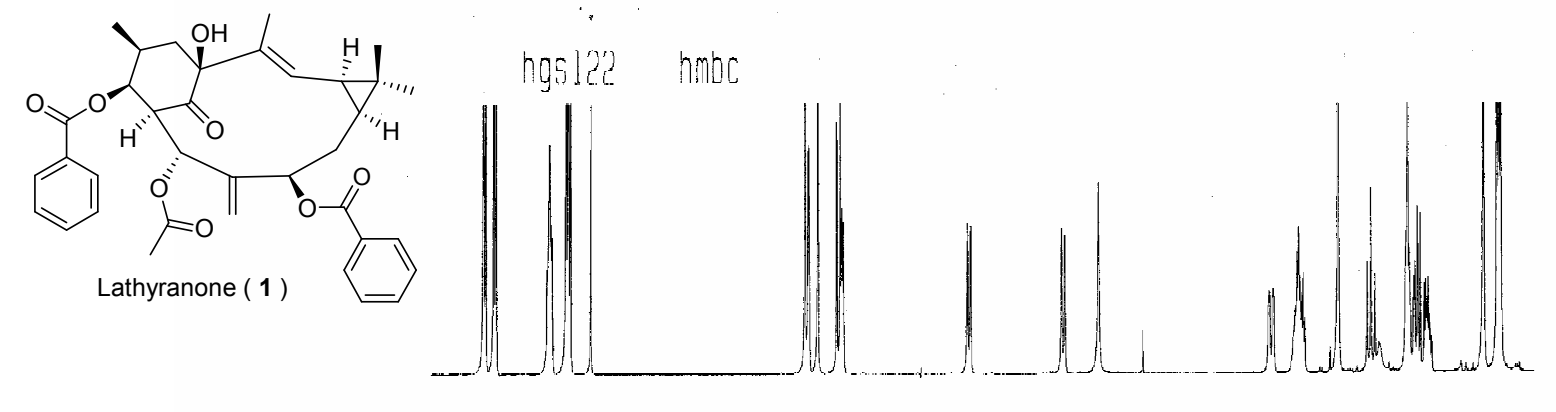

hoglet nmbe
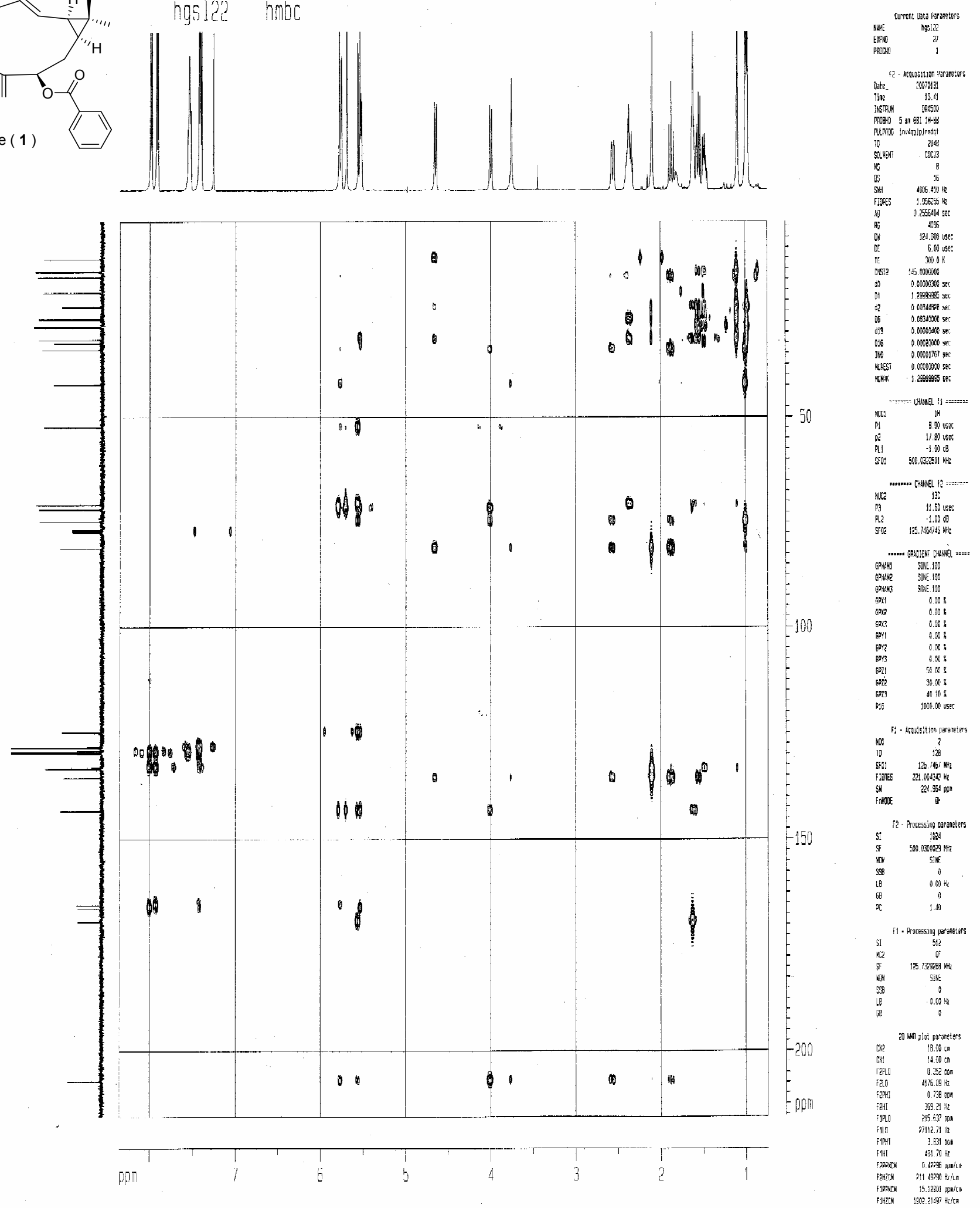
$1 \mathrm{H}-1 \mathrm{H}$ COSY spectrum of compound 1
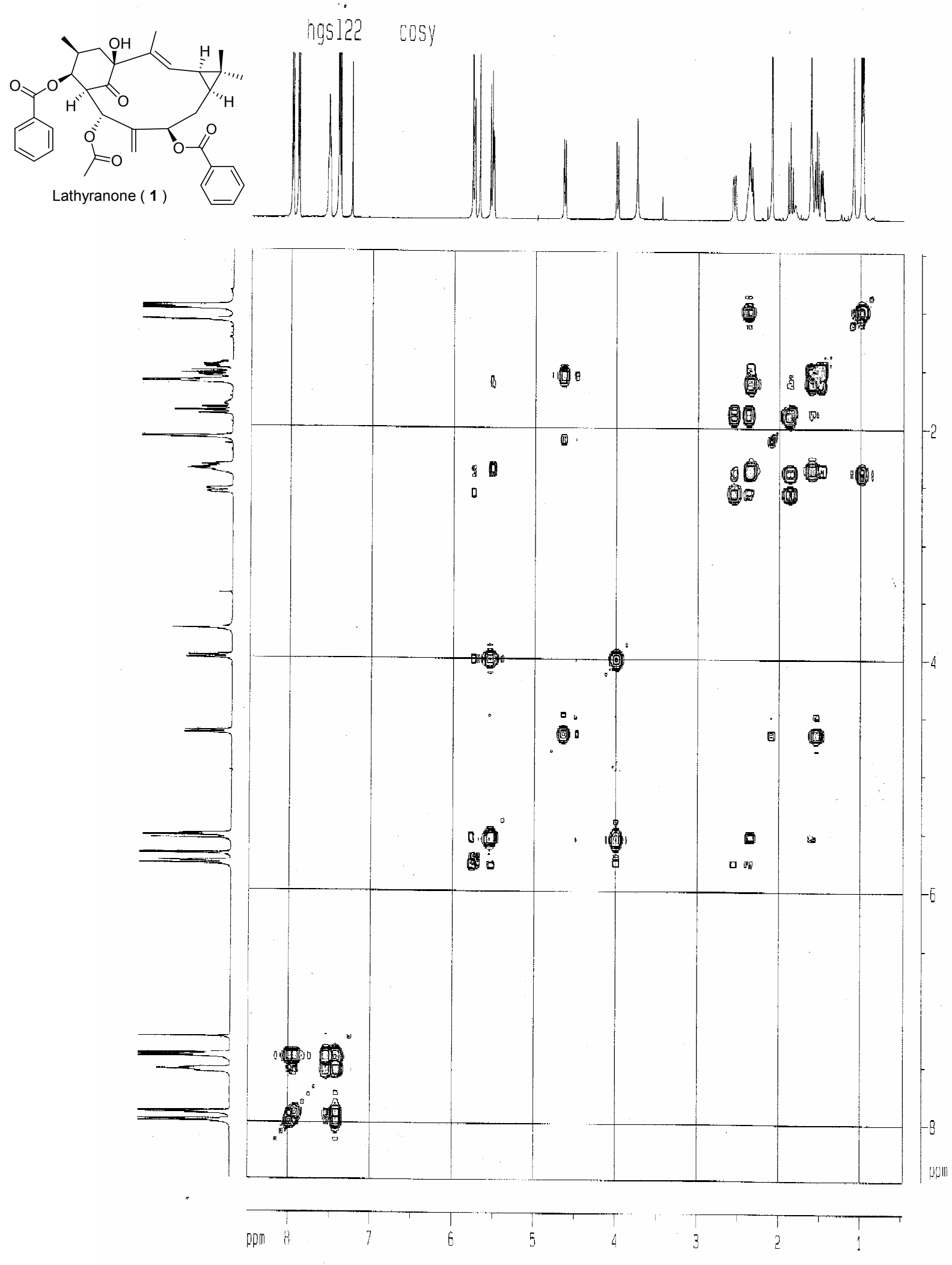

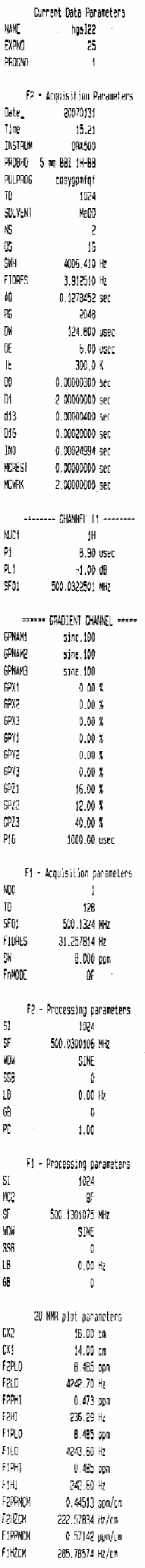


ROESY spectrum of compound 1

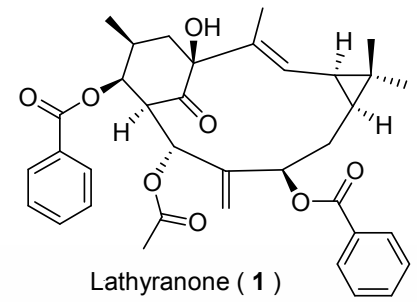

hosie2 roesy
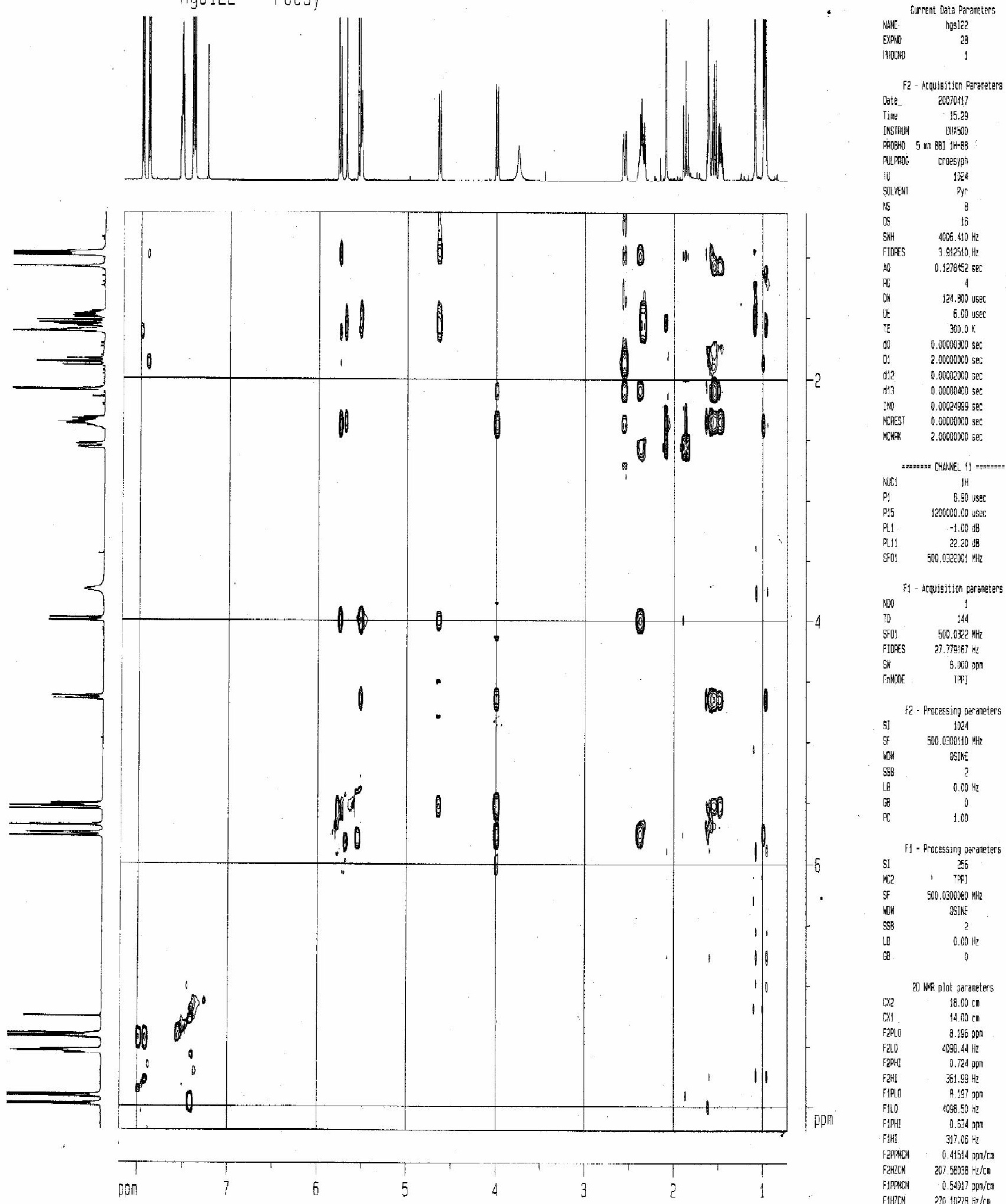$$
\text { mitis }
$$$$
1 \mathrm{H}
$$

100000

-1.60 . 18

22.20 .18

$500.03220091 .91 / 2$

F1- Acquisition perbateres

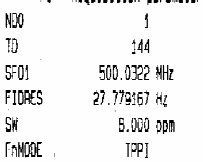

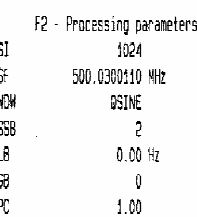

F1 - Procosting 0

\begin{tabular}{|c|c|}
\hline SI & 256 \\
\hline HOC2 & $1 \quad T P P]$ \\
\hline SF & 500,0300060 : Nht2 \\
\hline HOH & SSINE \\
\hline 598 & 2 \\
\hline LQ & $0.00 \mathrm{~Hz}$ \\
\hline 68. & 0 \\
\hline & 20 WW plot paraneters \\
\hline$[\times 2$ & $18.00 \mathrm{~cm}$ \\
\hline CX1. & $14.00 \mathrm{~cm}$ \\
\hline FEPLO & 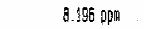 \\
\hline Fall & 4898.44 liz \\
\hline F2PHI & $0.724 \mathrm{ppm}$ \\
\hline F2HI & $361.99 \mathrm{~Hz}$ \\
\hline FIPLO & 8.197 ppm \\
\hline Fillo & $1008.50 \mathrm{nt2}$ \\
\hline FIPHI & 0.534 pap \\
\hline FIHI & $317,06 \mathrm{~Hz}$ \\
\hline IZPPACY & $0.41514 \mathrm{~g} 2 \mathrm{~g} / \mathrm{CA}$ \\
\hline FЕНСCK & $207.58838 \mathrm{~Hz} /$ C四 \\
\hline F.JPPYCH & $0.54917 \mathrm{ppag} / \mathrm{cm}$ \\
\hline FHACM & \\
\hline
\end{tabular}


